\title{
Características clínicas y demográficas de cáncer de colon, experiencia de 10 años
}

\section{- Pedro Ramos}

Oncocare, Bogotá, D.C.

Contacto: pramos152@yahoo.es

Introducción y objetivos. Evaluar las características clínicas y demográficas de cáncer de colon en una serie de pacientes seguidos durante 10 años.

Materiales y métodos. Se trata de un estudio observacional descriptivo, se registró la información de las características clínicas y demográficas de 624 pacientes con cáncer de colon, durante el período 2005-2014. Las pacientes fueron seguidas en intervalos cada 4 a 6 meses, en donde se documentaba el estado vital y la presencia de recaídas locales o sistémicas de la enfermedad. Se efectuaron análisis descriptivos.

Resultados. Se identificaron 624 pacientes con cáncer de colon, la edad promedio fue 63,10 años, del sexo femenino fue el $53,8 \%$. El estado clínico fue avanzado en el $71 \%$. El tipo histológico más frecuente fue adenocarcinoma clásico, el $75 \%$ recibió tratamiento adyuvante con más frecuencia, con esquema oxaliplatino-fluorouracilo-folinato-calcio (Folfox) y, para la enfermedad metastásica, el esquema más usual fue Folfox con o sin bevacizumab. La frecuencia de muerte fue del $23,8 \%$.

Conclusiones. En esta serie de pacientes la presentación clínica más frecuente fue estado avanzado y la quimioterapia usualmente más usada fue el esquema Folfox. La frecuencia de muertes fue del $23,8 \%$ en todo el tiempo de seguimiento.

\section{Manejo multimodal en adenocarcinoma gástrico metastásico}

\section{- Erwing Castillo Peñuela}

Hospital Manuel Uribe Ángel, Envigado (Antioquia).

Contacto: erwcastillo@yahoo.com

Introducción y objetivos. El adenocarcinoma gástrico es una patología frecuente en Colombia, siendo los estadios avanzados los más diagnosticados, con una sobrevida esperada menor a 12 meses.

Materiales y métodos. Paciente de 59 años con múltiples comorbilidades, en quien se diagnostica un adenocarcinoma gástrico con compromiso ganglionar, peritoneal y hepático. Recibió manejo con ECX por 3 ciclos y DCF por 6 ciclos, logrando respuesta imaginológica y endoscópica. Se llevó a gastrectomía parcial +D2, hepatectomía segmentaria y ablación con radiofrecuencia.

Resultados. Paciente en cuyo análisis de microscopia se documenta respuesta patológica completa hepática y casi total gástrica, logrando hasta el momento una sobrevida de 30 meses.

Conclusiones. La terapia multimodal debe ser una opción en neoplasias gástricas avanzadas, en pacientes seleccionados y basados en la respuesta a la quimioterapia previa. 